\title{
Fitase e digestibilidade aparente de nutrientes de rações por tilápias-do-nilo
}

\author{
Cláudio Luis Bock ${ }^{1}$, Luiz Edivaldo Pezzato², Osmar Ângelo Cantelmo ${ }^{3}$, Margarida Maria Barros ${ }^{4}$ \\ ${ }^{1}$ CEPTA/IBAMA, Pirassununga, SP. \\ 2 DMNA/FMVZ-UNESP, Botucatu, SP. \\ ${ }^{3}$ CEPTA/IBAMA, Pirassununga, SP. \\ ${ }^{4}$ DMNA/FMVZ-UNESP, Botucatu, SP.
}

RESUMO - Objetivou-se determinar a digestibilidade aparente de nutrientes de rações com diferentes níveis de fitase formuladas com alimentos de origem vegetal, em 90 juvenis de tilápia-do-nilo, pesando em média 120,0 g, alojados em nove tanques-rede (dez peixes/tanque). Adotou-se delineamento inteiramente casualizado, com nove tratamentos e seis repetições. Os tratamentos consistiram de uma ração suplementada com fósforo (controle) e de oito sem suplementação de fósforo, porém com 500, 1.000, 1.500, 2.000, 2.500, 3.000, 3.500 e 4.000 unidades de fitase (UF)/kg de ração, todas isoenergéticas e isoprotéicas. A inclusão de 1.500 UF melhorou a digestibilidade da MS e da EB e a disponibilidade de cálcio e fósforo das rações. O nível de 1.000 UF melhorou a disponibilidade de zinco e manganês.

Palavras-chave: fósforo, minerais, Oreochromis niloticus, tilápia

\section{Phytase and nutrient apparent digestibility of diets fed to Nile tilapia}

\begin{abstract}
The nutrient apparent digestibility of diet with increasing phytase levels based on vegetable ingredients fed to 90 Nile Tilapia juveniles averaging $120.0 \mathrm{~g}$ and allotted to none tanks (ten fishes/tank) was determined. The experiment was analyzed as a complete randomized design with nine treatments and six replicates. The treatments consisted of: control (with phosphorus supplementation) and eight treatments without phosphorus supplementation (with 500, 1,000, 1,500, $2,000,2,500,3,000,3,500$, and 4,000 units of phytase [UF]/kg of diet). All diets were formulated to be isoenergy and isoprotein. The 1,500 UF treatment increased DM and GE digestibility and dietary Ca and P availability. The 1,000 UF treatment increased $\mathrm{Zn}$ and Mn availability.
\end{abstract}

Key Words: mineral, Oreochromis niloticus, phosphorus, tilapia

\section{Introdução}

O crescimento e a intensificação da piscicultura nos últimos anos têm despertado o interesse sobre o impacto provocado ao ambiente. $\mathrm{O}$ fósforo e o nitrogênio são fundamentais na eutrofização de água doce. $\mathrm{O}$ fósforo, inclusive, tem sido considerado o principal nutriente que polui os ambientes aquaculturais. A criação de peixes enriquece o ambiente com os dejetos do metabolismo do alimento artificial, assim como dos fertilizantes orgânicos. Altos níveis de alimentação resultam em acúmulo de dejetos, o que altera o balanço do ecossistema, principalmente em razão da multiplicação do fitoplâncton, proporcional à quantidade de dejetos lançados.

Portanto, nutricionistas e fabricantes de rações para peixes têm se atentado à necessidade de dietas que satisfaçam, mas não excedam, às exigências de fósforo das espécies de interesse comercial. Têm-se estudado alterna- tivas para redução da excreção de fósforo das rações e minimização do impacto ambiental para aumento da produtividade zootécnica.

Os ingredientes mais utilizados nas rações contêm considerável concentração de fósforo. Entretanto, este mineral está presente nos vegetais na forma de fitato, indisponível aos peixes e demais animais monogástricos, pela ausência da enzima fitase. Portanto, a suplementação de fósforo inorgânico é necessária para obtenção do crescimento adequado dos peixes, o que acentua a excreção de fósforo, conduzindo à eutrofização do meio. A inclusão de fitase em rações contendo altos níveis de ingredientes vegetais pode reduzir a necessidade da adição de fósforo in orgânico às dietas e, conseqüentemente, a descarga desse mineral nos efluentes da piscicultura.

Nesse sentido, Forster et al. (1999) desenvolveram pesquisa com truta arco-íris (Oncorhynchus myskiss) e concluíram que a inclusão de 4.500 UF melhorou a qualidade 
nutritiva do concentrado protéico de canola e a disponibilidade de fósforo desse alimento, reduzindo sua liberação ao ambiente.

Vielma et al. (1998) realizaram, com a truta arco-íris, um estudo para avaliar a influência da inclusão dietética de fitase e de altos níveis de colecalciferol em rações formuladas com concentrado protéico de soja suplementadas com 0 e $1.500 \mathrm{UF} / \mathrm{kg}$ e $2.500,250.000$ e 2.500 .000 unidades internacionais de colecalciferol $/ \mathrm{kg}$. A inclusão de fitase melhorou a disponibilidade de fósforo, aumentando a concentração desse mineral, que foi significativamente mais alta nos ossos, no plasma e nos tecidos corporais.

Rodehutscord \& Pfeffer (1995) observaram, em trutas arco-íris, os efeitos da suplementação de fitase $(1.000 \mathrm{UF} / \mathrm{kg}$ de dieta) na disponibilidade do fósforo do concentrado protéico da soja. Embora as dietas contivessem elevada porcentagem de proteína de origem vegetal, a concentração do fósforo total foi otimizada em $60,0 \%$. Nesse mesmo sentido, Storebakken et al. (1998), em experimento com salmão do Atlântico (Salmo salar), avaliaram o concentrado protéico de soja como fonte de proteína dietética e concluíram que nenhum fitato foi encontrado nas dietas após o tratamento com fitase; que o concentrado protéico de soja foi equivalente à farinha de peixe como fonte de proteína dietética; que a inclusão de fitase resultou em melhora na digestibilidade da proteína, na conversão alimentar e na retenção de proteína e redução metabólica da excreção de nitrogênio; e que a disponibilidade de $\mathrm{Ca}, \mathrm{P}, \mathrm{Mg}$ e $\mathrm{Zn}$ foram maiores nas rações contendo fitase.

Gonçalves et al. (2004) desenvolveram pesquisa com juvenis (peso médio de 100,0 g) de tilápia-do-nilo (Oreochromis niloticus) para avaliar a ação da fitase em alimentos de origem vegetal. Segundo esse autor, a fitase agiu de forma diferenciada quando a suplementação de até $2.000 \mathrm{UF} / \mathrm{kg}$ não foi suficiente para melhorar a digestibilidade da MS, da proteína e da energia do milho extrusado, do farelo de trigo, do sorgo de baixo teor de tanino, da soja extrusada e do farelo de algodão. Entretanto, a inclusão de $1.000 \mathrm{UF} / \mathrm{kg}$ aumentou a digestibilidade da MS e da energia do milho, enquanto $2.000 \mathrm{UF} / \mathrm{kg}$ proporcionou essa resposta para os farelos de soja e de girassol. Segundo esses autores, a suplementação de até $2.000 \mathrm{UF} / \mathrm{kg}$ não aumentou a disponibilidade aparente de fósforo dos farelos de trigo e de algodão, mas a adição de $1.000 \mathrm{UF} / \mathrm{kg}$ promoveu aumento na disponibilidade do fósforo da soja extrusada e do farelo de girassol e a suplementação de $2.000 \mathrm{UF} / \mathrm{kg}$ proporcionou essa resposta para o farelo milho, o milho extrusado, o sorgo de baixo teor de tanino, o farelo de arroz, o farelo de soja e o glúten de milho.
Nesse contexto, realizou-se esta pesquisa com os objetivos de avaliar a disponibilidade de fósforo de rações formuladas com diferentes níveis de fitase para tilápia-donilo e verificar o efeito da inclusão de fitase na digestibilidade aparente dos nutrientes.

\section{Material e Métodos}

A pesquisa foi conduzida na Universidade Estadual Paulista - UNESP, no Laboratório de Nutrição de Organismos Aquáticos - AquaNutri da Faculdade de Medicina Veterinária e Zootecnia da Unesp, Campus de Botucatu.

As rações experimentais foram formuladas para ser isoenergéticas (3.200 kcal ED/kg de ração) e isoprotéicas $(30,0 \%$ PB). Foram testados nove tratamentos, um controle (ração com suplementação de fósforo em forma de fosfato bicálcico) e oito sem suplementação de fósforo, mas com inclusão de diferentes níveis de fitase $(500,1.000,1.500$, $2.000,2.500,3.000,3.500$ e $4.000 \mathrm{UF} / \mathrm{kg}$ de ração) $\left(\right.$ Natuphos $^{\circledR}$ 5000, BASF, Alemanha).

Como demonstrado nas Tabelas 1 e 2, as rações foram elaboradas com ingredientes energéticos e protéicos de origem vegetal. Para determinação dos coeficientes de digestibilidade aparente (CDA), as rações foram acrescentadas de $0,1 \%$ de óxido de cromo-III $\left(\mathrm{Cr}_{2} \mathrm{O}_{3}\right)$, conforme metodologia proposta por Graner (1972). Os ingredientes foram moídos a granulometria de 0,45 $\mathrm{mm}$ de diâmetro, sendo homogeneizados manualmente e peletizados com auxílio de equipamento peletizador (Ação científica). Posteriormente, os péletes foram desidratados em estufa com ventilação forçada a $55,0^{\circ} \mathrm{C}$ por 24 horas, sendo, então, fracionados a um diâmetro de $3 \times 5 \mathrm{~mm}$. As dietas foram mantidas em refrigeração a $4,0^{\circ} \mathrm{C}$.

Para obtenção das amostras e determinação dos coeficientes de digestibilidade e/ou disponibilidade aparente, foram utilizados nove aquários de alimentação confeccionados em fibra de vidro, de formato circular e capacidade de $250 \mathrm{~L}$, e cinco aquários para coleta de fezes, de formato cônico e capacidade de 300 L, também confeccionados em fibra de vidro.

Foram utilizados 90 juvenis de tilápia-do-nilo, pesando em média 120,0 g, alojados em nove tanques-rede (formato circular, confeccionados com tela plástica rígida, com malha de 1,5 cm entre nós e dimensões de 64,0 cm de altura por 52,0 cm de diâmetro) mantidos dentro de aquários de $250 \mathrm{~L}$, facilitando o manejo de alimentação e a coleta de fezes, conforme metodologia descrita por Pezzato et al. (2002).

Os peixes foram mantidos durante o dia nos aquários de alimentação, onde receberam quatro refeições até próximo 
Tabela 3 - Valores médios e desvio-padrão do coeficiente de digestibilidade aparente dos nutrientes (\%) das rações experimentais com diferentes níveis de fitase para tilápias-do-nilo

Table 3 - $\quad$ Average values and standard deviation of coefficient of nutrient apparent digestibity (\%) of the experimental diets with different phytase levels fed to Nile tilapia

\begin{tabular}{lccc}
\hline $\begin{array}{l}\text { Tratamento } \\
\text { Treatment }\end{array}$ & & $\begin{array}{c}\text { Digestibilidade } \\
\text { Digestibility }\end{array}$ & EB \\
& MS & PM & GE \\
\cline { 2 - 4 } & $77,87 \mathrm{~b}( \pm 1,75)$ & $9 P$ & $79,86 \mathrm{bc}( \pm 1,85)$ \\
\hline Com fosfato/Sem fitase & & $90,13( \pm 1,70)$ & $81,10 \mathrm{abc}( \pm 0,56)$ \\
With phosphate/without phytase & $78,82 \mathrm{ab}( \pm 1,15)$ & $92,79( \pm 1,34)$ & $80,63 \mathrm{bc}( \pm 1,25)$ \\
$500 \mathrm{UF} / \mathrm{kg}$ & $77,54 \mathrm{~b}( \pm 0,94)$ & $92,87( \pm 2,28)$ & $84,31 \mathrm{a}( \pm 0,95)$ \\
$1.000 \mathrm{UF} / \mathrm{kg}$ & $81,03 \mathrm{a}( \pm 1,44)$ & $95,15( \pm 1,07)$ & $81,10 \mathrm{abc}( \pm 0,86)$ \\
$1.500 \mathrm{UF} / \mathrm{kg}$ & $79,82 \mathrm{ab}( \pm 1,04)$ & $93,75( \pm 1,81)$ & $80,47 \mathrm{bc}( \pm 1,46)$ \\
$2.000 \mathrm{UF} / \mathrm{kg}$ & $77,74 \mathrm{~b}( \pm 0,7)$ & $92,95( \pm 1,89)$ & $78,18 \mathrm{c}( \pm 0,73)$ \\
$2.500 \mathrm{UF} / \mathrm{kg}$ & $73,84 \mathrm{c}( \pm 1,92)$ & $91,84( \pm 3,59)$ & $81,66 \mathrm{ab}( \pm 1,29)$ \\
$3.000 \mathrm{UF} / \mathrm{kg}$ & $79,65 \mathrm{ab}( \pm 1,19)$ & $94,53( \pm 1,15)$ & $80,25 \mathrm{bc}( \pm 1,38)$ \\
$3.500 \mathrm{UF} / \mathrm{kg}$ & $78,88 \mathrm{ab}( \pm 1,34)$ & $92,87( \pm 1,60)$ & \\
$4.000 \mathrm{UF} / \mathrm{kg}$ & & &
\end{tabular}

UF = unidades de fitase (Phytase units).

Valores com letras diferentes nas colunas indicam diferenças estatísticas significativas(Values with different letters in the columns indicate significant statistical differences, $P<0.01)$.

Tabela 4 - Valores médios e desvio-padrão da disponibilidade aparente de minerais das rações experimentais com diferentes níveis de fitase para tilápias-do-nilo

Table 4 - Average values and standard deviation of mineral apparent availability (\%) of the experimental diets with different phytase levels fed to Nile tilapia

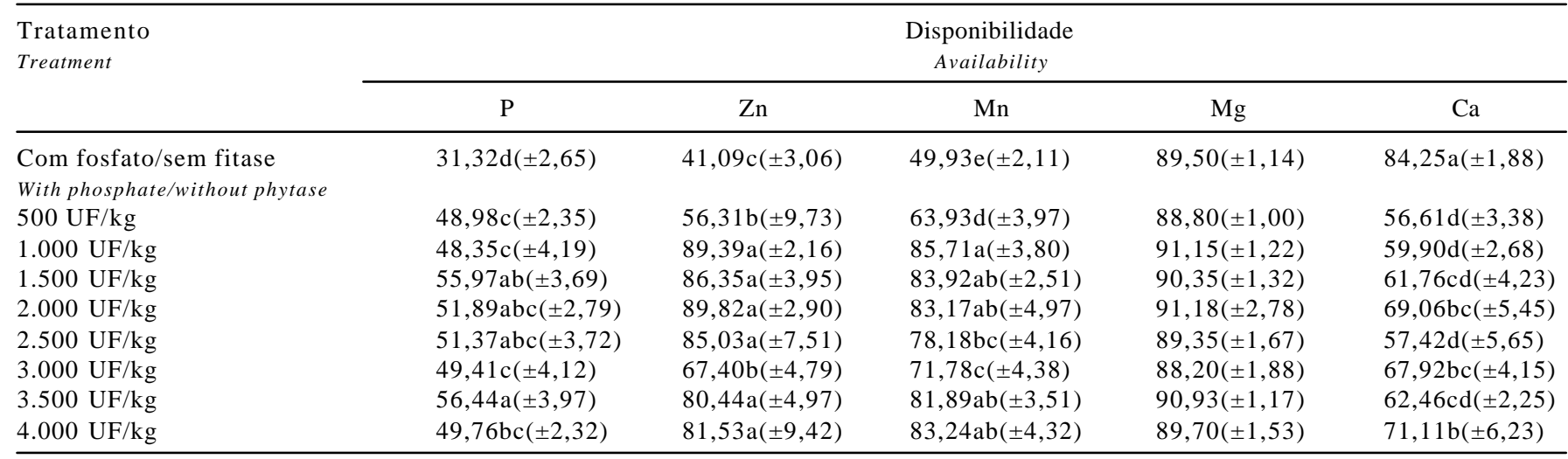

UF = unidades de fitase (Phytase units).

Valores com letras diferentes nas colunas indicam diferenças estatísticas significativas(Values with different letters in the columns indicate significant statistical differences, $P<0.01)$.

diferentes tratamentos (Tabela 3) indicaram diferenças $(\mathrm{P}<0,01)$ entre os tratamentos. Pelo teste de comparação de médias, verificou-se que a inclusão de fitase na ração, exceto no nível 3.000 UF/kg, resultou em coeficientes de digestibilidade da MS semelhantes ao obtido no tratamento controle, com suplementação de fósforo. Esse resultado comprovou que a fitase resultou em maior disponibilidade de fósforo para as atividades metabólicas dos peixes. Destaca-se ainda que o tratamento com $1.500 \mathrm{UF} / \mathrm{kg}$ proporcionou o melhor resultado para a digestibilidade aparente da MS, o que diverge dos resultados obtidos por Olívia-Teles et al. (1998), que, em estudo com seabass (Dicentrarchus labrax), observaram que a adição de 1.000 e $2.000 \mathrm{UF} / \mathrm{kg}$ não promoveu diferenças na digestibilidade desse nutriente. O efeito observado neste estudo pode ser explicado pela maior digestibilidade da MS do farelo de soja (Gonçalves et al., 2004) quando adicionadas $2.000 \mathrm{UF} / \mathrm{kg}$, observando-se que o farelo participou em $66,3 \%$ da ração utilizada nesta pesquisa. Entretanto, o tratamento com inclusão de $3.000 \mathrm{UF} / \mathrm{kgfoidiferente} \mathrm{dos}$ demais, com a menor média.

A inclusão dos diversos níveis de fitase assegurou coeficientes de digestibilidade da PB semelhantes ao observado no tratamento controle (Tabela 4). Entretanto, esses coeficientes de digestibilidade da PB não diferiram $(\mathrm{P}>0,01)$ entre os tratamentos, constatando-se apenas pequena tendência da enzima em melhorar os coeficientes de digestibilidade da proteína das rações. 
$\mathrm{O}$ tratamento $1.500 \mathrm{UF} / \mathrm{kg}$, a exemplo do ocorrido com a MS, apresentou o valor mais alto de digestibilidade da PB $(95,15 \%)$. Essa tendência em melhorar a digestibilidade da proteína deve ser considerada por sua importância às respostas zootécnicas e pela menor excreção de nitrogênio para o meio ambiente.

Estes resultados confirmam os encontrados para a carpa comum (Cyprinus carpio) por Shäfer et al. (1995), utilizando dietas com farinha de peixe e farelo de soja. Esses autores não notaram diferenças nos CDA com a inclusão de $500 \mathrm{UF} / \mathrm{kg}$. Oliva-Teles et al. (1998), ao utilizarem o farelo de soja em substituição à farinha de peixe $(65,6 \%$ da proteína dietética) com suplementação de 1.000 e $2.000 \mathrm{UF} / \mathrm{kg}$, também não verificaram diferenças significativas entre os tratamentos. Storebakken et al. (1998), avaliando dietas com substituição parcial da farinha de peixe por concentrado protéico de soja com a inclusão de 5.000 UF, também obtiveram coeficientes de digestilidade semelhantes.

Submetendo os resultados dos coeficientes de digestibilidade da EB (Tabela 3) ao teste de comparação de médias, observou-se que, na ração com $1.500 \mathrm{UF} / \mathrm{kg}$, houve significativa melhora na digestibilidade da EB e que, nos demais níveis de inclusão, não foram encontradas diferenças. Houve exceção, conforme observado para os coeficientes de digestibilidade da MS e da PB, no tratamento com $3.000 \mathrm{UF} / \mathrm{kg}$.

Em trabalhos realizados anteriormente com peixes (Oliva-Teles et al., 1998; Shäfer et al., 1995; Forster et al., 1999), não foram relatadas diferenças para a digestibilidade aparente de energia com o seabass, a carpa comum e a truta arco-íris, respectivamente. Thompson \& Yoon (1984), citados por Gonçalves et al. (2004), a partir de estudos realizados com frangos de corte, relataram que, no estado nativo, o fitato pode se complexar com o amido. Ravindran (1999), citado por Gonçalves et al. (2004), relatou que a digestão do amido pode ser negativamente influenciada pelo ácido fítico. Por outro lado, Selle et al. (1997), citados por Gonçalves et al. (2004), informaram que algumas rações à base de alimentos de origem vegetal foram avaliadas com sucesso utilizando-se a enzima fitase como suplemento para o aumento do coeficiente de digestibilidade da EB. Entre esses alimentos, destacaram-se os farelos de soja e de trigo, os quais compuseram a dieta utilizada nesta pesquisa.

Pela análise de variância dos resultados (Tabela 4), observaram-se diferenças significativas $(\mathrm{P}<0,01)$ entre os tratamentos para os coeficientes de disponibilidade aparente dos minerais. A análise pelo teste de comparação de médias revelou que a adição de fitase nas rações elevou significativamente a disponibilidade de fósforo em todos os níveis de inclusão, diferenciando-os do tratamento com suplementação de fósforo (controle).

Conforme pode ser observado na Tabela 4 , a presença de fitase nas rações melhorou $(\mathrm{P}<0,05)$ a disponibilidade do fósforo. Entre os níveis testados, o de $1.500 \mathrm{UF} / \mathrm{kg}$ pode ser considerado o mais adequado para essa prática.

Segundo Forster et al. (1999), a disponibilidade de fósforo dietético foi maior com a inclusão de $4.500 \mathrm{UF} / \mathrm{kg}$. Shäfer et al. (1995) verificaram melhora de mais de 50,0\% no coeficiente de absorção aparente do fósforo ao utilizarem $500 \mathrm{UF} / \mathrm{kg}$, semelhante aos resultados obtidos nesta pesquisa. Hughes \& Soares Jr. (1998) encontraram altas diferenças no coeficiente de disponibilidade aparente do fósforo nos tratamentos com 800, 1.300 e $2.400 \mathrm{UF} / \mathrm{kg}$, comparados ao controle, sem inclusão de fitase, e também obtiveram melhores resultados com o maior nível de inclusão da fitase. Rodehutscord \& Pfeffer (1995) também relataram que a disponibilidade de fósforo foi de $25,0 \%$ na dieta não suplementada, ao passo que a suplementação de fitase aumentou a disponibilidade de $\mathrm{P}$ para $57,0 \%$.

A utilização de fitase promoveu melhora significativa na disponibilidade de Zn e Mn em relação ao tratamento sem suplementação. Os melhores coeficientes de disponibilidade foram obtidos com níveis iguais ou superiores a $1.000 \mathrm{UF} / \mathrm{kg}$ para ambos os minerais.

Como demonstrado na Tabela 4, a presença de fitase não resultou em aumento na disponibilidade do magnésio e não foi efetiva na disponibilização de cálcio. Por outro lado, apenas na ração controle (com suplementação de fósforo) utilizou-se esse nível de fitase, o que pode explicar os resultados obtidos nos demais tratamentos, nos quais a quantidade de cálcio pode ter sido inferior à exigência dessa espécie e ter influenciado as respostas obtidas.

Os resultados obtidos para cálcio demonstraram significativa redução na disponibilidade desse mineral quando comparados aos da dieta com suplementação de fósforo. Entretanto, quando comparados os níveis de inclusão de fitase, os tratamentos com 2.000, 3.000 e $4.000 \mathrm{UF} / \mathrm{kg}$ promoveram melhora $(\mathrm{P}<0,05)$, observando-se maior dis ponibilidade de cálcio nos níveis mais elevados de fitase. A redução da disponibilidade de cálcio das rações com a inclusão de fitase, em comparação àquela suplementada com fosfato, pode, possivelmente, ser atribuída ao fato de que a suplementação foi realizada na forma de $4,0 \%$ de fosfato bicálcico.

Vielma et al. (1998), trabalhando com truta arco-íris e inclusão de $1.500 \mathrm{UF} / \mathrm{kg}$, observaram que a disponibilidade de fósforo e cálcio melhorou significativamente com a suplementação de fitase à ração. Segundo os autores, a suplementação não influenciou a absorção aparente de 
Mg, Mn e Zn e o aumento de fósforo dietético e dos níveis de cálcio pode reduzir a disponibilidade de zinco em peixes, como observado por Hardy \& Shearer (1985) e Satoh et al. (1992).

Storebakken et al. (1998), em pesquisa com salmão do Atlântico, substituíram a farinha de peixe pelo concentrado de soja tratado com $5.000 \mathrm{UF} / \mathrm{kg}$. Esses autores observaram que os coeficientes de digestibilidade aparente de $\mathrm{Ca}$ e $\mathrm{Mg}$ elevaram significativamente com a inclusão da fitase. Segundo os autores, os coeficientes de digestibilidade aparente desses minerais foram reduzidos pela ação do fitato da dieta contendo o concentrado protéico de soja. O coeficiente de disponibilidade aparente do magnésio na dieta composta pelo concentrado protéico de soja + $5.000 \mathrm{UF} / \mathrm{kg}$ foi semelhante ao obtido nesta pesquisa.

Segundo Hilton (1989) e Gatlin III \& Phillips (1989), a concentração de zinco em peixes alimentados com dietas contendo soja é reduzida como resultado da diminuição do coeficiente de disponibilidade aparente do zinco causada pela combinação deste mineral $(\mathrm{Zn})$ com o fitato. Essa afirmativa foi confirmada pelos resultados de disponibilidade obtidos nesta pesquisa (Tabela 4), na qual a utilização da fitase melhorou significativamente sua disponibilidade em relação ao tratamento sem suplementação, obten do-se os melhores coeficientes com níveis a partir de $1.000 \mathrm{UF} / \mathrm{kg}$.

\section{Conclusões}

Em rações para tilápias-do-nilo formuladas exclusivamente com ingredientes de origem vegetal, a inclusão de $1.500 \mathrm{UF} / \mathrm{kg}$ é suficiente para disponibilizar fósforo e cálcio e permitir adequado coeficiente de digestibilidade aparente da MS e da energia, sendo necessária a inclusão de $1.000 \mathrm{UF} / \mathrm{kg}$ para adequada disponibilidade de zinco e manganês.

\section{Agradecimento}

À indústria Supremais Produtos Bioquímicos Ltda., pelo apoio científico. Aos Departamentos de Química e de Bromatologia da FZEA - USP - Campus de Pirassununga, SP. Ao Laboratório de Bromatologia do CEPTA/IBAMA Pirassununga, SP.

\section{Literatura Citada}

AMERICAN PUBLIC HEALTH ASSOCIATION - ALPHA. Standard methods: for the examination of water and wastewater. 15.ed. Washington, D.C.: ALPHA, 1980. 1134p.
ASSOCIATION OF OFFICIAL AGRICULTURAL CHEMISTS AOAC. Official methods of analysis of the Association of Official Agricultural Chemists. 14.ed. Washington, D.C.: 1984. $1141 \mathrm{p}$.

BOYD, C.E. Water quality management for pond fish culture Amsterdam: Elsevier Scientific Publishing, 1982. 730p.

FOSTER, I.; HIGGS, D.A.; DOSANJH, B.S. et al. Potential for dietary phytase to improve the nutritive value of canola protein concentrate and decrease phosphorus output in rainbow trout (Oncorhynchus mykiss) held in $11^{\circ} \mathrm{C}$ fresh water. Aquaculture, v.179, p.109-125, 1999.

GATLIN-III, D.D.; PILLIPS, H.F. Dietary calcium, phytase and zinc interactions in channel catfish. Aquaculture, v.79, p.259266, 1989.

GONÇALVES, G.S.; PEZZATO, L.E.; BARROS, M.M. et al. Digestibilidade aparente e suplementação de fitase em alimentos vegetais para tilápia do Nilo. Acta Scientiarum, v.26, n.3, p.313-321, 2004.

GRANER, C.A.F. Determinação do crômio pelo método colorimétrico da s-difenilcabazida. Botucatu: Universidade Estadual Paulista, 1972. 112p. Tese (Doutorado em Ciências) Universidade Estadual Paulista, 1972.

HARDY, R.W.; SHEARER, K.D. Effect of dietary calcium phosphate an zinc supplementation on whole body zinc concentration of rainbow trout (Salmo gairdneri). Canadian Journal of Fisheries and Aquatic Sciences, v.42, p.181-184, 1985.

HILTON, J.W. The interaction of vitamins, minerals and diet composition in the diet of fish. Aquaculture, v.79, p.223224, 1989.

HUGHES K.P.; SOARES JR., J.H. Efficacy of phytase on phosphorus utilization in practical diets fed to striped bass Morone saxatilis Aquaculture Nutrition, v.4, p.133-140, 1998.

MIRANDA, E.C.; PEZZATO, A.C.; PEZZATO, L.E. et al. Disponibilidade aparente de fósforo em ingredientes pela tilápia do Nilo (Oreochromis niloticus). Acta Scientiarium, v.22, n.3, p.669-675, 2000.

OLIVA-TELES, A.; PEREIRA, J.P.; GOUVEIA, A. et al. Utilization of diets supplemented with microbial phytase by seabass. Aquatic Living Resources, v.11, n.4, p.255-259, 1998.

PEZZATO, L.E.; MIRANDA, E.C.; BARROS, M.M. et al. Digestibilidade aparente de ingredientes pela tilápia do Nilo (Oreochromis niloticus). Revista Brasileira de Zootecnia, v.31, n.4, p.1595-1604, 2002.

RODEHUTSCORD M.; PFEFFER, E. Effects of supplemental microbial phytase on phosphorus digestibility and utilization in rainbow trout (Oncorhynchus mykiss). Water Science \& Technology, v.31, n.10, p.143-147, 1995.

SATOH, S.; IZUME, K.; TAKEUCHI, T. Effect of supplemental tricalcium phosphate on zinc and manganese availability to common carp. Nippon Suisan Gakkaishi, v.58, n.3, p.539$545,1992$.

SHÄFER, A.; KOPPE, W.M.; MEYER-BURGDORFF, H.G. et al. Effects of microbial phytase on utilization of native phosphorus carp in a diet based on soybean meal. Water Science \& Technology, v.31, n.10, p.140-155, 1995.

STOREBAKKEN, T.; SHEARER, K.D.; ROEM, A.J. Availability of protein, phosphorus and other elements in fish meal, soyprotein concentrate and phytase-treated soy-proteinconcentrate-based diets to Atlantic salmon. Aquaculture v.161, p.365-379, 1998.

VIELMA J.; LALL, S.P.; KOSKELA, J. et al. Effects of dietary phytase and cholecalciferol on phosphorus bioavailability in rainbow trout (Oncorhynchus mykiss). Aquaculture, v.163, p.309-323, 1998 . 